09

\title{
Возбуждение и ионизация частицы в одномерной потенциальной яме нулевого радиуса предельно коротким световым импульсом
}

\author{
(C) P.М. Архипов ${ }^{1,2}$, М.В. Архипов ${ }^{1}$, А.В. Пахомов ${ }^{1}$, Н.Н. Розанов ${ }^{1,2}$ \\ ${ }^{1}$ Санкт-Петербургский государственный университет, \\ 199034 Санкт-Петербург, Россия \\ ${ }^{2}$ ФТИ им. А.Ф. Иофре РАН, \\ 194021 Санкт-Петербург, Россия \\ e-mail: arkhipovrostislav@gmail.com, m.arkhipov@spbu.ru, antpakhom@gmail.com, nnrosanov@mail.ru
}

Поступила в редакцию 24.11.2021 г.

В окончательной редакции 24.11.2021 г.

Принята к публикации 03.12.2021 г.

С помощью приближения внезапных возмущений Мигдала решается задача о возбуждении и ионизации частицы в одномерном потенциале нулевого радиуса предельно коротким импульсом. В такой одномерной дельтаобразной потенциальной яме имеется всего один энергетический уровень. Показано, что при длительностях импульса, меньших характерного периода осцилляций волновой функции частицы в связанном состоянии, населенность уровня (и вероятность ионизации) определяется отношением электрической площади импульса к характерной „мере“ площади, обратно пропорциональной области локализации частицы в связанном состоянии.

Ключевые слова: униполярные импульсы, электрическая площадь импульса, атомная мера площади.

DOI: $10.21883 /$ OS.2022.03.52171.2959-21

\section{Введение}

В последние годы активно изучается возможность получения униполярных электромагнитных импульсов с ненулевой электрической площадью, определяемой как

$$
S_{E} \equiv \int_{-\infty}^{\infty} E(t) d t
$$

$E(t)$ - напряженность электрического поля в заданной точке пространства (см. обзор [1] и цитируемую литературу). Такие импульсы могут иметь множество различных применений, например, для сверхбыстрого и эффективного управления динамикой волновых пакетов в веществе, по сравнению с обычными биполярными импульсами, ускорения зарядов и др. приложений [1].

Как показывают результаты различных исследований, воздействие униполярных импульсов на микрообъекты определяется электрической площадью импульса, а не его энергией, если длительность импульса меньше характерного периода осцилляций волнового пакета в веществе [2-7]. Некоторые способы экспериментального определения униполярности излучения и его электрической площади были предложены впервые сравнительно недавно [8].

В случае, когда длительность возбуждающего импульса короче периода осцилляций волнового пакета в веществе, стандартная теория фотоионизации Келдыша становится неприменимой [7], и определение некоторых физических величин, например, параметра Келдыша, задающего критерий сильного и слабого поля, требует пересмотра.
Для характеристики степени воздействия униполярных импульсов на квантовые объекты недавно была введена новая физическая величина - атомная мера площади, обратно пропорциональная характерному размеру системы [4]. Как показано в этой работе, населенность основного состояния простейших многоуровневых квантовых систем (атом водорода, квантовый осциллятор и др.) определяется отношением электрической площади импульса к ее атомной мере.

В последующей работе была рассмотрена ионизация трехмерных квантовых систем (атом водорода, сферическая квантовая точка, трехмерный потенциал нулевого радиуса) предельно коротким импульсом [7]. Было показано, что вероятность ионизации также определяется отношением электрической площади импульса к ее атомной мере, которая обратно пропорциоанльна характерному размеру системы в основном состоянии.

Теоретическое описание взаимодействия предельно коротких и униполярных импульсов с многоуровневыми квантовыми системами является сложной задачей. Для моделирования реальных квантовых систем привлекательной является модель потенциала нулевого радиуса. Эта модель активно используется для изучения различных процессов в ядерной и атомной физике, описания поведения ионов во внешних полях, см. [9-11] и приведенную литературу.

Задача ионизации из трехмерной $\delta$-ямы циркулярно поляризованной монохроматической волной была рассмотрена в [12-14]. В работе [15] трехмерная модель потенциала нулевого радиуса была применена для 
анализа вырывания электрона из отрицательных ионов униполярными импульсами.

Наиболее простой является модель потенциала нулевого радиуса в одномерном случае. Несмотря на ее простоту, она также была использована для моделирования различных систем, таких как водороподобные атомы, двухатомные молекулы, ионы и более сложные системы [16-19].

В ряде работ рассматривались задачи взаимодействия мощного лазерного излучения с атомными системами, которые моделировались одномерным потенциалом нулевого радиуса [20-22]. Подробнее о применении данной модели в различных задачах см. обзор [23] и цитируемую в нем литературу.

Как уже отмечалось выше, недавно было показано, что вероятность ионизации широкого класса трехмерных квантовых систем предельно короткими импульсами определяется отношением электрической площади импульса к введенной недавно величине мере площади, которая обратно пропорциональной характерному размеру системы [7].

В настоящей работе изучается вероятность сохранения связанного состояния и ионизации частицы, находящейся в одномерной потенциальной яме нулевого радиуса под действием предельно короткого импульса длительностью короче периода осцилляций волновой функции частицы в связанном состоянии.

Произведено сравнение со случаем трехмерного потенциала нулевого радиуса. Показано, что вероятности сохранения связанного состояния в одномерном и трехмерном случаях сильно отличаются по форме. Однако и в одномерном случае можно ввести характерную меру площади $S_{0}$, обратно пропорциональную области локализации электрона в связанном состоянии.

\section{Теоретическое рассмотрение и обсуждение результатов}

Уравнение Шредингера с дельтаобразным потенциалом в одномерном случае имеет вид

$$
\begin{gathered}
\psi^{\prime \prime}+\frac{2 m}{\hbar^{2}}(E-U(x)) \psi=0, \\
U(x)=-V_{0} \delta(x) .
\end{gathered}
$$

В такой яме имеется всего один уровень энергии $E=-\frac{m}{2 \hbar^{2}} V_{0}^{2}$. Нормированные волновые функции связанного состояния даются выражением [24]

$$
\begin{gathered}
\psi_{0}(x)=\sqrt{\alpha} e^{\alpha x}, \quad x<0, \\
\psi_{0}(x)=\sqrt{\alpha} e^{-\alpha x}, \quad x>0, \\
\alpha \equiv \frac{m}{\hbar^{2}} V_{0} .
\end{gathered}
$$

В этих выражениях присутствует характерный размер области локализации электрона

$$
x_{0}=\frac{1}{2 \alpha}=\frac{\hbar^{2}}{2 m V_{0}} \text {. }
$$

Длительность импульса возбуждения $\tau$ предполагается короче периода осцилляций волновой функции в связанном состоянии, $\tau \ll T=2 \pi \hbar / E$. Например, в случае иона $\mathrm{H}^{-}$, для которого модель потенциала нулевого радиуса активно используется, время $T=5.4 \mathrm{fs}$ в трехмерном рассмотрении [15]. Поэтому оптические импульсы аттосекундной длительности могут активно быть использованы в подобных задачах [25-27].

Волновая функция частицы после импульса в приближении внезапных возмущений Мигдала имеет известный вид $[6,7,28]$

$$
\Psi_{+}(x)=\psi_{0}(x) e^{-i \frac{q}{\hbar} S_{E} x},
$$

где $q$ - заряд частицы.

Амплитуда связанного состояния после импульса определяется выражением

$$
a_{0}=\int_{-\infty}^{\infty} \psi_{0}^{2}(x) e^{-i \frac{q}{\hbar} S_{E} x} d x .
$$

Проводя интегрирование, из выражений (2) и (4) можно найти населенность связанного состояния частицы в яме после окончания действия импульса:

$$
w_{0}=\left|a_{0}\right|^{2}=\frac{1}{\left(1+S_{E}^{2} / S_{0}^{2}\right)^{2}} .
$$

Населенность связанного состояния в (5) убывает с ростом электрической площади по крайней мере как $S_{E}^{-4}$. В этом выражении введена „мера“ площади $S_{0} \equiv \frac{2 \alpha \hbar}{q}=\frac{\hbar}{q x_{0}}$, обратно пропорциональная характерному размеру области локализации электрона $x_{0}$. Она имеет смысл характерного значения электрической площади импульса, при которой возможно эффективное опустошение связанного состояния системы.

Таким образом, населенность определяется отношением электрической площади импульса к ее характерной мере $S_{E} / S_{0}$. Соответственно вероятность ионизации $w_{\text {ion }}=1-w_{0}$ также определяется электрической площадью импульса с характерной мерой $S_{0}$, обратно пропорциональной размеру системы $x_{0}$.

В случае же трехмерного потенциала нулевого радиуса выражение для вероятности сохранения связанного состояния по форме отличается от выражения в одномерном случае (5) и имеет вид арктангенса $[7,15]$ :

$$
\begin{gathered}
w_{0}=a_{00}^{2}, \quad w_{\text {ion }}=1-w_{0}, \\
a_{00}=\left(S_{0} / S_{E}\right) \arctan \left(S_{E} / S_{0}\right) .
\end{gathered}
$$

Однако и в трехмерном случае стоящая в этом выражении характерная мера площади $S_{0}$ также обратно пропорциональна области локализации электрона в связанном состоянии в яме [7].

Отметим сходство (5) с вероятностью сохранения основного состояния атома водорода [7,29]:

$$
w_{0}=\left[1+\left(S_{E} / S_{a t}\right)^{2}\right]^{-4}
$$


Только в данном случае вероятность убывает быстрее, как $S_{E}^{-8}$. А величина атомной меры площади $S_{a t}=\frac{2 \hbar}{q a_{0}}$ также обратно пропорциональна радиусу первой боровской орбиты $a_{0}$, т. е. характерному размеру системы.

\section{Заключение}

Таким образом, в случае одномерной модели потенциала нулевого радиуса вероятность сохранения связанного состояния частицы и ее ионизации определется отношением электрической площади импульса к ее характерной мере, $S_{E} / S_{0}$. Эта мера площади $S_{0}$ обратно пропорциональна характерному размеру области локализации частицы в связанном состоянии $x_{0}$.

Понятие меры площади, введенное впервые в [4,7], справедливо для широкого класса квантовых систем как одномерных, так и трехмерных. Им можно пользоваться для оценки значения электрической площади импульса, необходимого для эффективного возбуждения и ионизации квантовых систем с помощью униполярных и субцикловых импульсов.

Величину меры площади необходимо принимать во внимание при анализе взаимодействия предельно коротких импульсов с квантовыми объектами, когда длительность импульса короче характерного периода осцилляций волновой функции в квантовой системе.

\section{Финансирование работы}

Исследование выполнено при финансовой поддержке РНФ в рамках научного проекта 21-72-10028.

\section{Конфликт интересов}

Авторы заявляют, что у них нет конфликта интересов.

\section{Список литературы}

[1] Р.М. Архипов, М.В. Архипов, Н.Н. Розанов. Квант. электрон.; [R.M. Arkhipov, M.V. Arkhipov, N.N. Rosanov, Quant. Electron., 50 (9), 801 (2020)].

[2] P.H. Bucksbaum. AIP Conf. Proc., 323 (1), 416 (1994).

[3] D. Dimitrovski, E.A. Solov'ev, J.S. Briggs. Phys. Rev. A, 72, 043411 (2005).

[4] Р.М. Архипов, М.В. Архипов, А.В. Пахомов, Н.Н. Розанов. Письма в ЖЭТФ, 114 (3), 156 [R.M. Arkhipov, M.V. Arkhipov, A.V. Pakhomov, N.N. Rosanov. JETP Lett., 114 (3), 129 (2021)].

[5] Р.М. Архипов, М.В. Архипов, И. Бабушкин, А.В. Пахомов, Н.Н. Розанов. Письма в ЖЭТФ, $114(5), 298$ (2021). [R.M. Arkhipov, M.V. Arkhipov, A.V. Pakhomov, N.N. Rosanov. JETP Lett., 114 (5), 250 (2021)].

[6] R.M. Arkhipov, M.V. Arkhipov, I. Babushkin, A. Demircan, U. Morgner, N.N. Rosanov. Opt. Lett., 44 (5), 1202 (2019).

[7] N. Rosanov, D. Tumakov, M. Arkhipov, R. Arkhipov. Phys. Rev. A, 104, 063101 (2021).
[8] М.В. Архипов, А.Н. Цыпкин, М.О. Жукова, А.О. Исмагилов, А.В. Пахомов, Н.Н. Розанов, Р.М. Архипов. Письма в ЖЭТФ, $115(1), 3$ (2022). [M.V. Arkhipov, A.N. Tsypkin, M.O. Zhukova, A.O. Ismagilov, A.V. Pakhomov, N.N. Rosanov, R.M. Arkhipov, JETP Lett., 115(1) (2022). DOI: $10.1134 / \mathrm{S} 0021364022010015]$.

[9] E. Fermi. Ric. Sci., 7, 13 (1936).

[10] Ю.Н. Демков, В.Н. Островский. Метод потенциалов нулевого радиуса в атомной бизике (Изд-во ЛГУ, 1975); [Y.N. Demkov, V.N. Ostrovskii. Zero-Range Potentials and Their Applications in Atomic Physics (Plenum, New York, 1988)].

[11] N.L. Manakov, M.V. Frolov, B. Borca, A.F. Starace. J. Phys. B, 36, R49 (2003).

[12] I.J. Berson. J. Physics B: Atomic and Molecular Physics, 8 (18), 3078 (1975).

[13] Н.Л. Манаков, Л.П. Рапопорт. ЖЭТФ, 69 (3), 842 (1075). [N.L. Manakov, L.P. Rapoport. Sov. Phys. JETP, 42 (3), 430 (1976)].

[14] Н.Б. Делоне, В.П. Крайнов. Атом в сильном световом поле (Атомиздат, М., 1978).

[15] T.P. Grozdanov, J. Jaćimović. Phys. Rev. A, 79, 013413 (2009).

[16] A.A. Frost. J. Chem. Phys., 25, 1150 (1956).

[17] A.A. Frost. J. Chem. Phys., 22, 1613 (1954).

[18] A.A. Frost, F.E. Leland. J. Chem. Phys., 25, 1154 (1956).

[19] I. Lapidus. Am. J. Phys., 38, 905 (1970).

[20] S. Geltman. J. Physics B, 10 (5), Article 019, 831 (1977).

[21] S. Geltman. J. Physics B, 27, 1497 (1994).

[22] Q. Su, B.P. Irving, C.W. Johnson, J.H. Eberly. J. Physics B: Atomic, Molecular and Optical Physics, 29 (23), 5755 (1996).

[23] M. Belloni, R.W. Robinett. Physics Reports, 540 (2), 25 (2014).

[24] N. Zettili. Quantum Mechanics Concepts and Applications (John Wiley \& Sons, 2009).

[25] M.T. Hassan, T.T. Luu, A. Moulet, O. Raskazovskaya, P. Zhokhov, M. Garg, N. Karpowicz, A.M. Zheltikov, V. Pervak, F. Krausz, E. Goulielmakis. Nature, 530, 66 (2016).

[26] H.-C. Wu., J. Meyer-ter-Vehn. Nature Photon., 6, 304 (2012).

[27] J. Xu, B. Shen, X. Zhang, Y. Shi, L. Ji, L. Zhang, T. Xu, W. Wang, X. Zhao, Z. Xu. Sci. Rep., 8, 2669 (2018).

[28] А.Б. Мигдал. ЖЭТФ, 9, 1163 (1939).

[29] Н.Н. Розанов. Опт. и спектр., $124(1), 75$ (2018); [N.N. Rosanov. Opt. Spectrosc., 124 (1), 72 (2018)]. 